\title{
Simulation aéraulique d'une cellule climatisée Comparaison entre calculs - N3S version 3.1 et mesures sur maquette
}

\author{
D. Manzoni, F.-X. Rongère
}

EDF-DER, Service Réacteurs nucléaires et échangeurs, Transferts thermiques et aérodynamiques, 6, quai Watier, Chatou

\section{II $\square$ INTRODUCTION}

Plus encore qu'en chauffage, les mouvements d'air au sein d'une pièce climatisée sont des facteurs déterminants de performance énergétique et de confort. En effet, premièrement, l'émetteur de froid, contrairement à un émetteur de chaleur, doit lutter contre la convection naturelle pour irriguer la pièce. L'étendue de la zone de confort et les gradients enregistrés sont donc directement liés à la qualité de son soufflage. Deuxièmement, les occupants d'un bâtiment sont beaucoup plus sensibles aux vitesses locales d'air froid que d'air chaud du fait de l'écart de température plus important avec leur organisme. Ces vitesses font partie de l'indice de confort de la norme internationale applicable à la climatisation [1].

Cette étude a donc pour objectif de compléter la qualification du logiciel N3S pour les études d'aéraulique des bâtiments initiée en 1992 et 1993 sur les questions de chauffage. On s'intéresse en particulier à la représentation qualitative des écoulements afin d'évaluer l'utilisation du code pour la mise au point de modèles zonaux. Les résultats quantitatifs sont également discutés dans le but de qualifier N3S pour une utilisation directe dans le cadre d'une analyse de conditions de confort en complément de ces modèles.

La configuration simulée est un écoulement bidimensionnel induit par un jet froid vertical associé à une reprise d'air au voisinage du sol. Elle est représentative du fonc- tionnement d'un ventilo-convecteur ou d'un climatiseur autonome dans une pièce d'habitation ou un bureau. Cette configuration a fait l'objet également d'une expérimentation réalisée au Laboratoire d'Etudes Thermiques de Poitiers. Le banc d'essais est équipé d'un ensemble métrologique (anémométrie laser, tomographie laser, mesures de température par thermocouples fins) permettant la visualisation des écoulements et des mesures locales instantanées de vitesses et de température [2].

On présente dans un premier temps les caractéristiques géométriques et thermohydrauliques de l'expérience. On s'attache ensuite à la modélisation N3S du problème, et à l'interprétation des résultats numériques. Ces résultats sont analysés sous deux aspects :

- la comparaison qualitative et quantitative calculs/ expériences,

- l'étude de l'influence du profil d'entrée sur les dimensions du jet et l'irrigation de la cellule d'essai.

\section{II ÉTUDE EXPÉRIMENTALE}

\subsection{Description}

Le Laboratoire d'Etudes Thermiques de Poitiers (L.E.T.) a mis au point une maquette représentative d'une pièce d'habitation ou de bureau climatisée [2]. La cellule est

Aerothermal analysis of a refrigerated room

\author{
Comparison between numerical simulation by N3S issue 3.1 and experimental data
}

This report presents the comparison between numerical simulation performed with the N3S issue 3.1 CFD code and experimental data, for a typical bidimensionnal configuration of a room refrigerated by a fan-coil unit.

In particular, the qualitative representation of the flows is examined in order to evaluate the use of the code for the implementation of zonal models. The quantitative results are also discussed in order to qualify N3S issue 3.1 for a direct utilization within the analysis of the comfort conditions. 


\section{SYMBOLES ET NOTATIONS}

\begin{tabular}{|c|c|c|}
\hline Symbole & Unité & Signification \\
\hline $\begin{array}{l}C \\
D \\
e \\
E \\
\text { Fr } \\
g \\
h \\
H \\
H p \\
k \\
L \\
m \\
Q \\
Q e \\
Q s \\
R e \\
s \\
t \\
T \\
T_{0} \\
T_{\text {init }} \\
T_{\text {mil }} \\
T_{p} \\
T_{j} \\
T e \\
T s \\
V \\
U_{j} \\
\alpha \\
\beta \\
\varepsilon \\
\lambda_{p} \\
\mu \\
v \\
\rho \\
\rho s \\
\rho e \\
\rho 0\end{array}$ & 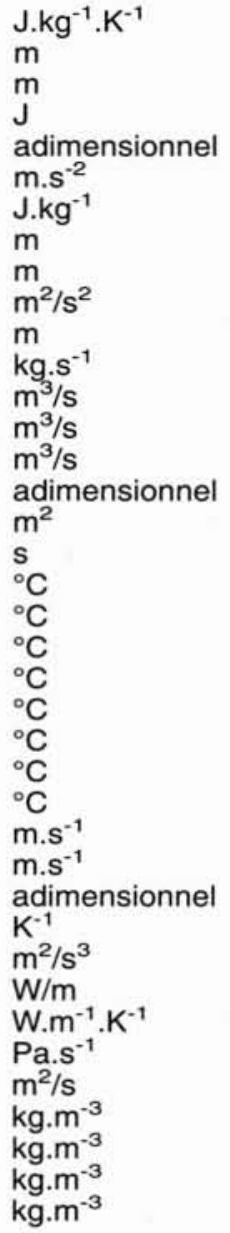 & 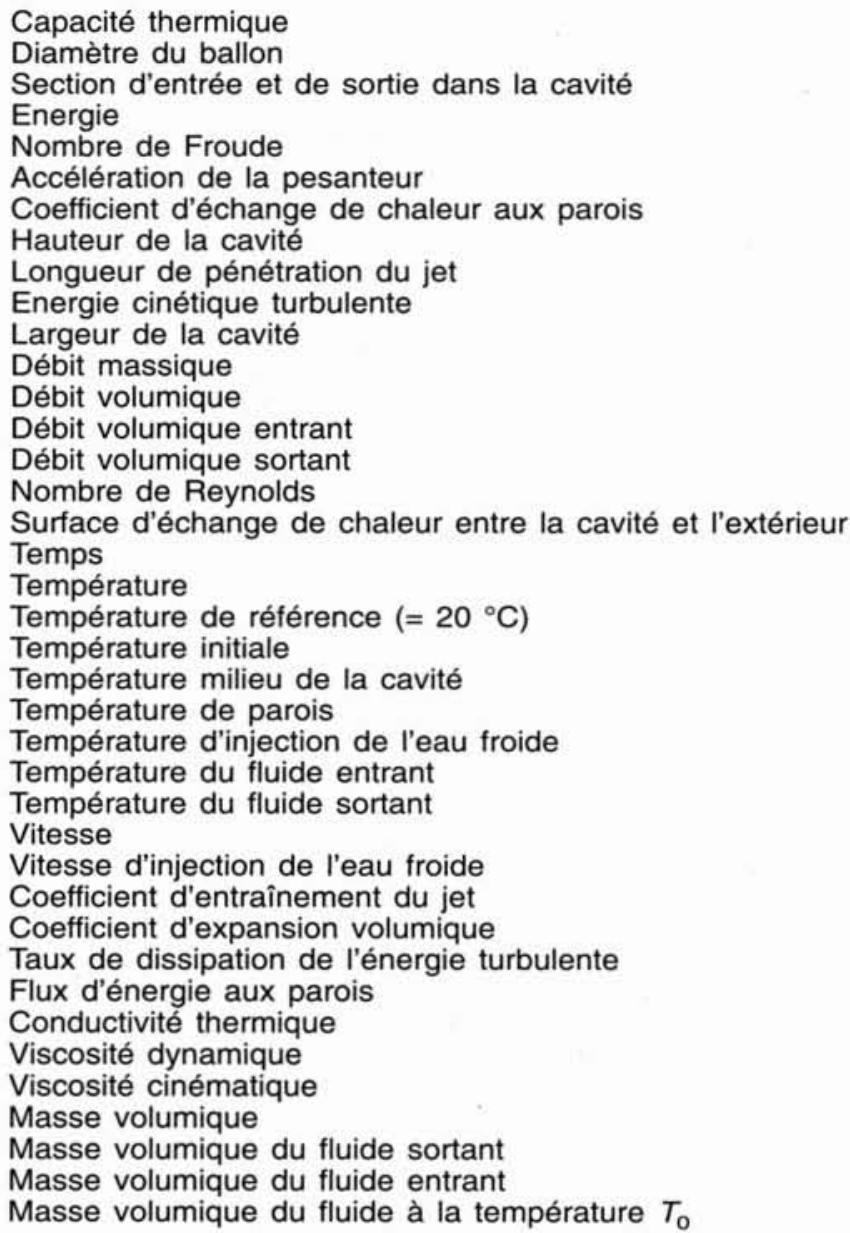 \\
\hline
\end{tabular}

identique à celle utilisée lors des essais en chauffage [2], dont la géométrie a été adaptée à la climatisation [1], [3], [4], [5].

La maquette est réalisée en plexiglass. Un soin particulier a été apporté au contrôle des transferts. Les mesures ont été effectuées point par point, à l'aide d'un laser pour la vitesse et de deux thermocouples disposés à l'extrémité d'un système de déplacement automatique pour la température.

La configuration géométrique est schématisée sur la figure 1:

$H=L=1,04 \mathrm{~m}$. La largeur de la cellule est de $1 \mathrm{~m}$.

La section d'entrée est égale à la section de sortie. Son épaisseur vaut : $e=23 \mathrm{~mm}$, et sa largeur est égale à celle de la cellule d'étude.

L'épaisseur de la séparation entrée/sortie est de : $4 \mathrm{~mm}$, et sa hauteur de $10 \mathrm{~mm}$. Ce dispositif schématise une unité intérieure de climatisation ou un ventilo-convecteur.

La configuration étudiée est bidimensionnelle.

\subsection{Conditions aux limites}

Les quatre parois sont à la même température $T_{p}=26,7^{\circ} \mathrm{C}$.
Le jet d'entrée est entièrement défini par ses débits massique, enthalpique et de quantité de mouvement. Leur connaissance passe par la détermination des profils de vitesse et de température dans la section de sortie de la bouche d'entrée. Le profil de vitesse mesuré est donné par la figure 2.

La valeur moyenne de la vitesse d'entrée du jet est: $U_{j}=U_{\text {moy }}=0,73 \mathrm{~m} / \mathrm{s}$.

La température moyenne du jet à l'entrée est: $T_{j}=17^{\circ} \mathrm{C}$.

Les conditions d'injections sont caractérisées par les nombres de Reynolds et de Froude :

$$
\mathrm{Re}=\frac{U_{j} \cdot e}{v} \text { et } \mathrm{Fr}=\frac{U_{j}}{\sqrt{g \cdot \beta \cdot e \cdot\left(T_{p}-T_{j}\right)}} .
$$

D'après les données expérimentales, on obtient :

$$
\operatorname{Re}=1090 \text { et } \mathrm{Fr}=8,5
$$

\subsection{Résultats expérimentaux}

Les profils horizontaux de vitesse verticale, les profils verticaux de vitesse horizontale et les profils de température sont représentés à la fin de cette note, en compa- 
$\mathrm{L}$

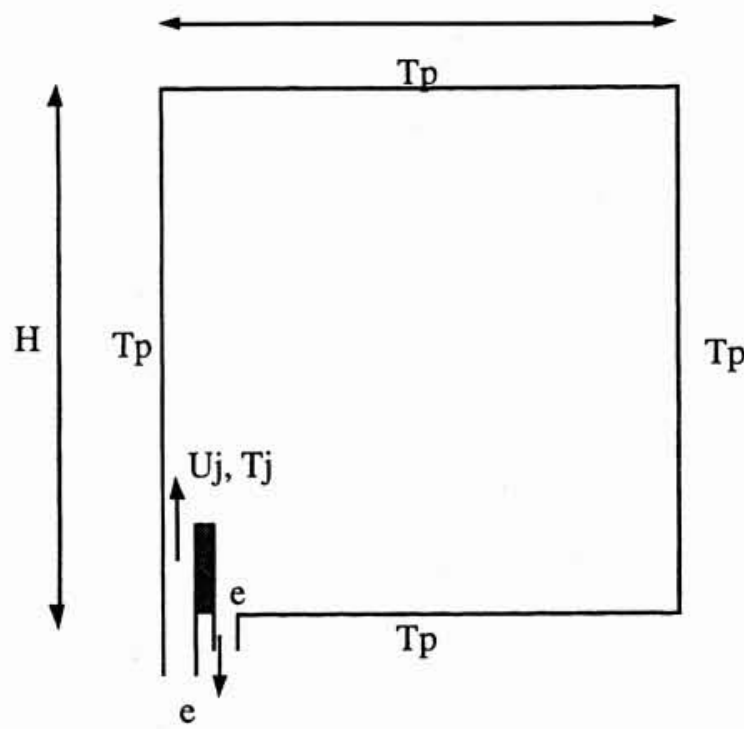

1. Géométrie de la cavité.

Uy $(\mathrm{m} / \mathrm{s})$

ㅁ $\quad$ y $(m / s)$

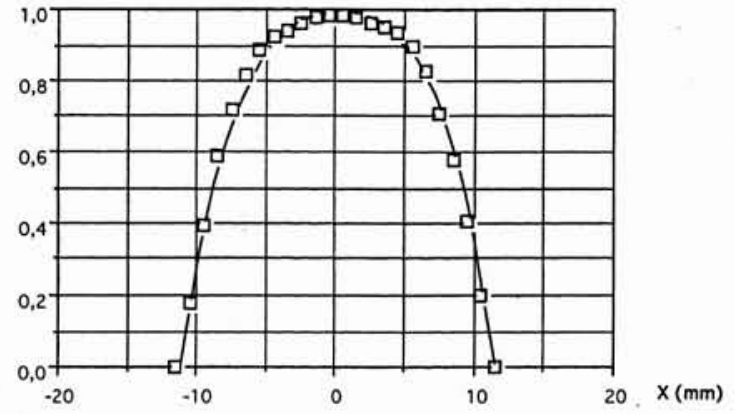

$\left(y=0.99325+4.9436 .10^{-4} \cdot x-3,0876.10^{-3} x^{2}-1,9354.10^{-6} x^{3}-3.53424 .10^{-5} \cdot x^{4} R^{2}=0.996\right)$

\section{Profil de vitesse en sortie de la bouche d'entrée.}

raison avec les profils numériques. On visualise également les champs de température et de vitesse sur les figures 3 et 4 .

D'après le champ de vitesse on constate que le jet d'air froid induit un tourbillon dans la partie gauche de la cavité, tournant dans le sens des aiguilles' d'une montre, régi par les forces d'inertie, et un tourbillon tournant dans le sens contraire dans la partie droite de la cavité, régi par les forces de flottabilité et de viscosité.

D'après le champ de température, on constate que la partie gauche correspondant au jet, est bien refroidie (entre $17^{\circ} \mathrm{C}$ et $21^{\circ} \mathrm{C}$ ), alors que la partie droite profite moins de l'air frais mais sa température est plus homogène : elle vaut $22,8^{\circ} \mathrm{C}$
Notons que des mesures complémentaires pour la vitesse dans des plans parallèles situés à $5 \mathrm{~cm}$ de part et d'autre du plan habituel de mesure ont été réalisées de façon à déceler d'éventuels effets 3D. Les résultats obtenus ont montré que ces effets restent faibles et localisés aux frontières du jet. On peut donc considérer l'écoulement comme 2D. Plus de cinq cents points de mesures ont été réalisés, dont certains ont été doublés.

On remarquera enfin une légère dispersion des résultats de température dans la partie haute de la cavité. Cette dispersion est due à l'instabilité de l'écoulement dans cette région où se rencontre le jet et le mouvement général de l'air dans la cavité.

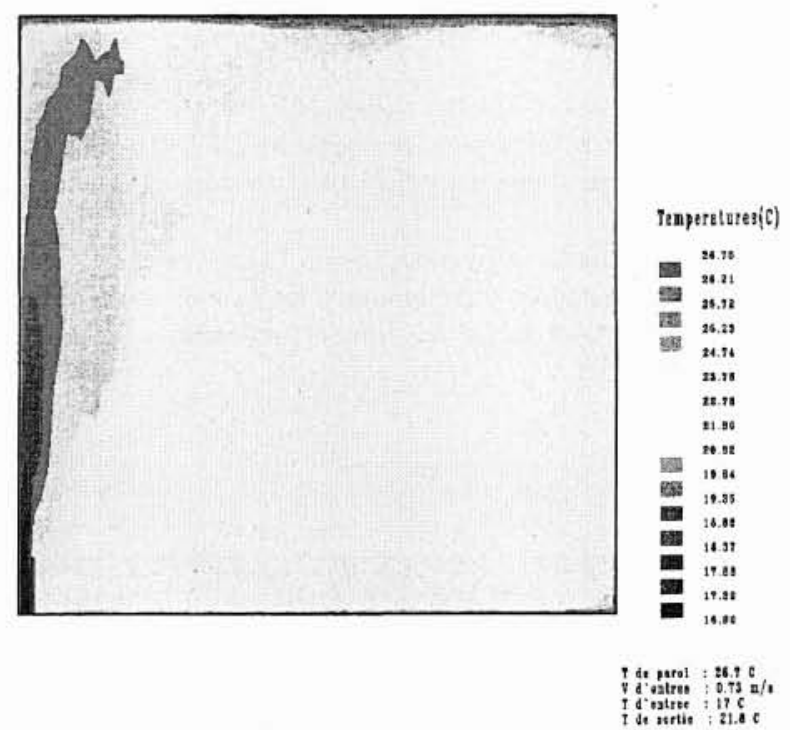

3. Champ de température expérimental.

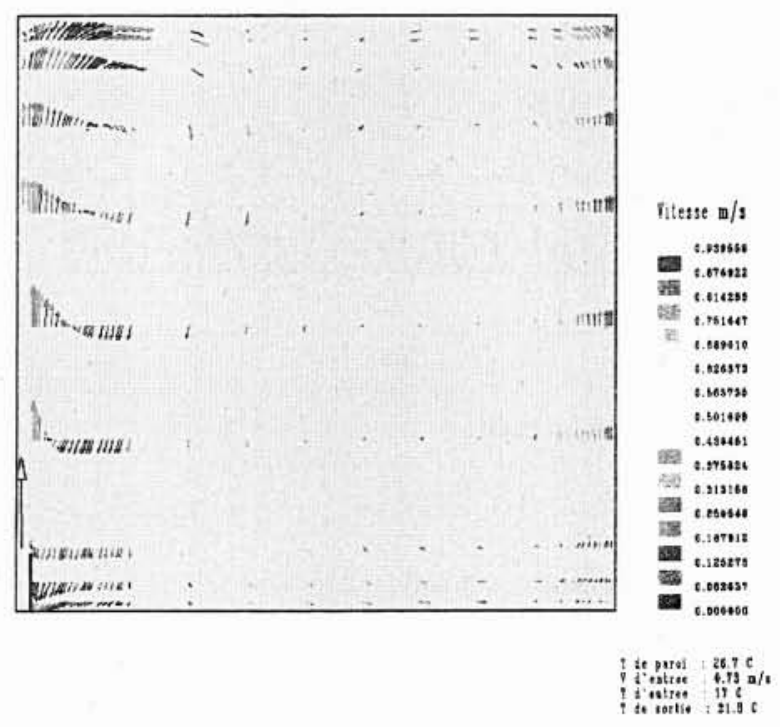

4. Champ de vitesse expérimental. 


\section{III —ÉTUDE NUMÉRIQUE}

\subsection{Description}

Le maillage P1-isoP2 est composé de 1557 éléments et de 3342 nœuds P2 (fig. 5). Ce maillage est plus fin près de l'arrivée et de la sortie d'air et des parois. Il reste cependant relativement grossier au centre de la cavité. On compte 7 nœuds sur l'entrée et 5 nœuds sur la sortie. Ces nœuds sont équi-répartis.

La géométrie de la cavité modélisée est identique aux données expérimentales (fig. 1).

\subsection{Conditions aux limites}

Conformément aux conditions expérimentales, on a choisi :

La température de parois : $T_{p}=26,7^{\circ} \mathrm{C}$.

Le jet d'air froid a pour température : $T_{j}=17^{\circ} \mathrm{C}$ et a pour vitesse moyenne $U_{j}=0,73 \mathrm{~m} / \mathrm{s}$ sur tous les nœuds d'entrée.

Pour une vitesse moyenne $U_{j}=0,73 \mathrm{~m} / \mathrm{s}$ du jet, il est possible de pondérer différemment les nœuds à l'entrée, tout en conservant la même vitesse moyenne.

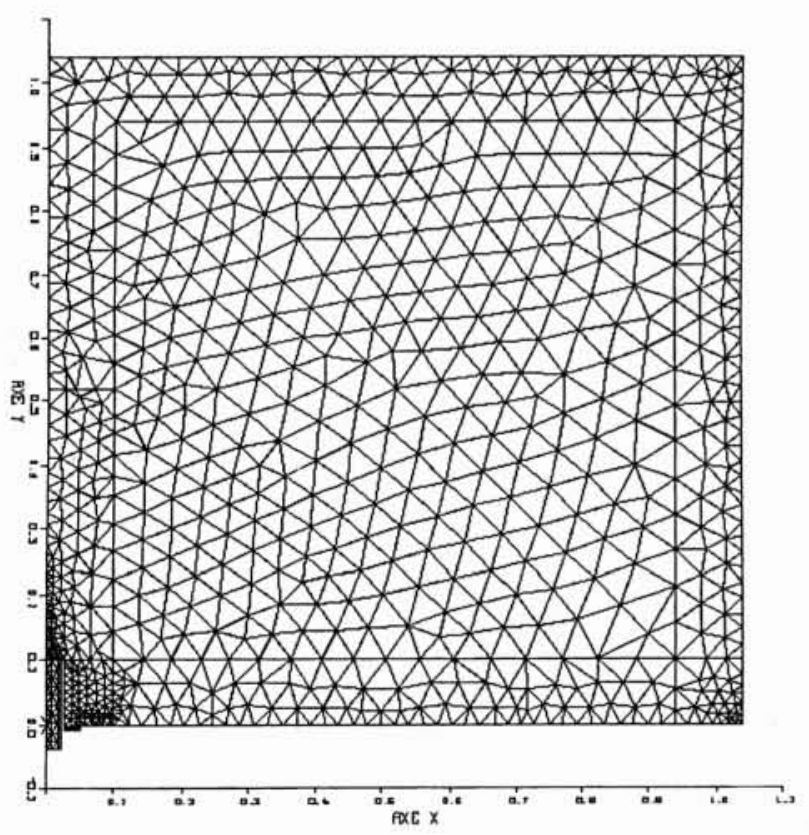

5. Maillage de la cavité.

L'entrée comporte 7 nœuds :

\begin{tabular}{|l|c|c|c|c|c|c|c|}
\hline $\mathrm{N}^{\circ}$ nœud & 580 & 2560 & 581 & 2562 & 582 & 2564 & 583 \\
\hline $\begin{array}{l}\text { abscisse } \\
(\mathrm{m})\end{array}$ & 0,0 & 0,0038 & 0,0076 & 0,0115 & 0,0153 & 0,0191 & 0,023 \\
\hline
\end{tabular}

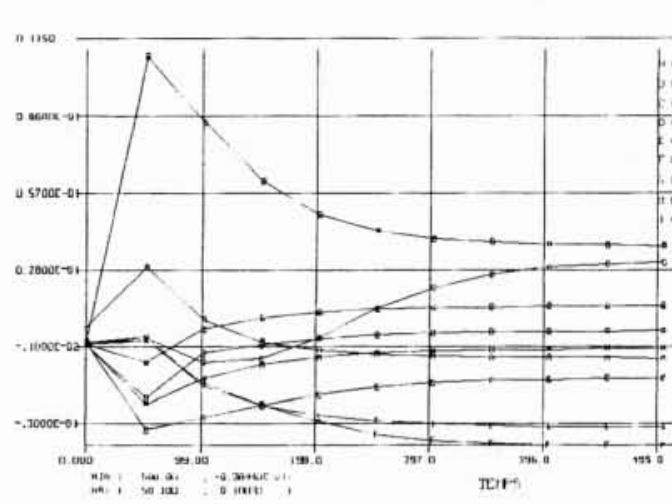

mtstoriaut

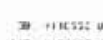

bi. Nituse

1.... intent

ises, vitessc 1

Hat, viresse 1

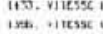

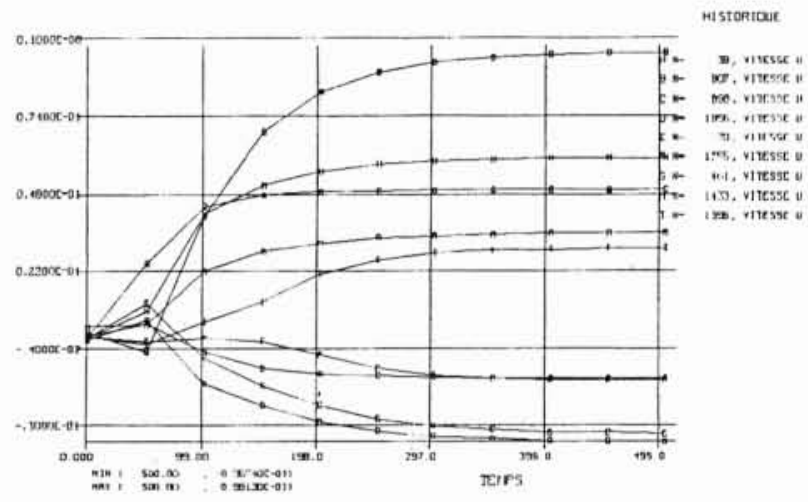

Mistoribue

MISTDFIDUE

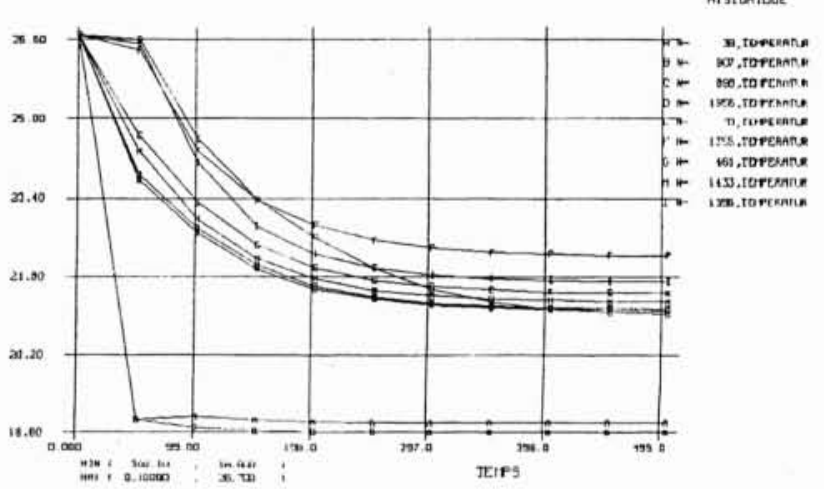

6. Historiques de température et de vitesse pour le cas 1 .

Trois profils de vitesse ont donc été testés pour évaluer l'incidence de la forme du jet sur l'écoulement dans la cellule :

CAS 1 : Profil expérimental (fig. 2)

\begin{tabular}{|l|c|c|c|c|c|c|c|}
\hline$N^{\circ}$ nœud & 580 & 2560 & 581 & 2562 & 582 & 2564 & 583 \\
\hline vitesse $U_{j}(\mathrm{~m} / \mathrm{s})$ & 0,0 & 0,76 & 0,95 & 0,99 & 0,95 & 0,76 & 0,0 \\
\hline
\end{tabular}

\section{CAS 2 : Profil plat}

\begin{tabular}{|l|c|c|c|c|c|c|c|}
\hline$N^{\circ}$ nœud & 580 & 2560 & 581 & 2562 & 582 & 2564 & 583 \\
\hline vitesse $U_{j}(\mathrm{~m} / \mathrm{s})$ & 0,73 & 0,73 & 0,73 & 0,73 & 0,73 & 0,73 & 0,73 \\
\hline
\end{tabular}


CAS 3: Profil plat au milieu du jet

\begin{tabular}{|l|c|c|c|c|c|c|c|}
\hline $\mathrm{N}^{\circ}$ nœud & 580 & 2560 & 581 & 2562 & 582 & 2564 & 583 \\
\hline vitesse $U_{j}(\mathrm{~m} / \mathrm{s})$ & 0,1 & 0,64 & 1,0 & 1,0 & 1,0 & 0,64 & 0,1 \\
\hline
\end{tabular}

Les valeurs de ces trois cas ont été choisies de telle sorte que la vitesse moyenne $U_{i}=0,73 \mathrm{~m} / \mathrm{s}$ soit conservée.

La turbulence est prise en compte à l'aide du modèle $k-\varepsilon$ standard. Les données en entrée de type Dirichlet pour $k$ et $\varepsilon$ sont fixées classiquement par:

$$
k=1,5 \cdot\left(5 \% \cdot U_{j}\right)^{2} \quad \text { et } \quad \varepsilon=\frac{k}{10 \cdot \Delta t}
$$

on impose donc sur les nœuds à l'entrée :

$k=2.10^{-3} \mathrm{~m}^{2} / \mathrm{s}^{2} \quad$ et $\varepsilon=2.10^{-3} \mathrm{~m}^{2} / \mathrm{s}^{3}$

Les grandeurs caractéristiques du fluide sont: $\mu=1,8510^{-5} \mathrm{~kg} / \mathrm{m} . \mathrm{s}$

$$
\begin{aligned}
\mathrm{v} & =1,5710^{-5} \mathrm{~m}^{2} / \mathrm{s} \\
C_{p} & =1006 \mathrm{~J} / \mathrm{kg} \cdot \mathrm{K} \\
\lambda & =0,0262 \mathrm{~W} / \mathrm{m} \cdot \mathrm{K} .
\end{aligned}
$$

La variation de la masse volumique est simulée dans N3S par l'option « Rho variable»:

$$
\rho=\rho_{0}\left[1-\beta\left(T-T_{0}\right)\right]
$$

avec $T_{0}=20{ }^{\circ} \mathrm{C}, \rho_{0}=1,205 \mathrm{~kg} / \mathrm{m}^{3}$ et $\beta=3.410^{-3} \mathrm{~K}^{-1}$ Notons que cette loi correspond au développement limité d'ordre I de la loi des gaz parfaits, en supposant la pression constante.

La résolution du problème de Stokes est effectuée par la méthode de Chorin.

\subsection{Analyse des résultats}

\subsubsection{Comparaisons calculs/expériences}

Dans ce chapitre, seule la comparaison des mesures avec les calculs du cas 1 (profil expérimental) est présentée. Elle est complétée au paragraphe 3.3.2. par la mise en regard des résultats numériques obtenus pour les différents profils d'injection.

Pour ce calcul 5000 itérations ont été effectuées avec un pas de temps de 0.1 seconde CPU, ce qui correspond à $3000 \mathrm{~s}$ CPU environ sur le cray Y-MP, soit $0.18 \mathrm{~s} / 1000$ næuds et par pas de temps. Le régime permanent est obtenu à l'aide d'une intégration transitoire, 4000 itérations au minimum sont nécessaires pour y parvenir ( $\mathrm{cf}$. historiques fig. 5).

Notons que pour un pas de temps de $0,1 \mathrm{~s}$ les nombres de Courant (1) maximum et minimum sont respectivement égaux à 27.7 et $5.10^{-4}$ environ. La région des nombres de

(1) Soient $V$ la vitesse du fluide, $\Delta x$ la dimension caractéristique des mailles considérées et $\Delta t$ le pas de temps, le nombre de Couran est égal à $V \cdot \Delta t / \Delta x$.
Courant élevés est limitée au voisinage immédiat de l'entrée et de l'extraction d'air où de petits éléments sont associés à des vitesses élevées. Au centre de la cavité, les éléments étant environ dix fois plus grands, le nombre de Courant maximum est de 2,7 .

La figure 7 visualise le champ de température et de vitesse pour le cas I. Qualitativement, l'écoulement est correctement représenté. Conformément aux résultats expérimentaux, on remarque dans la partie gauche de la cavité un tourbillon dans le sens des aiguilles d'une montre régi par les forces d'inertie, et dans la partie droite un tourbillon inverse régi par les forces de flottabilité et de viscosité. On note toutefois que les dimensions du jet froid sont surestimées par N3S.

Les comparaisons quantitatives calculs/mesures sont détaillées ci-dessous sur la base :

- des profils verticaux de vitesse horizontale,

- des profils horizontaux de vitesse verticale,

- des profils de température, présentés figures 8 à $/ 1$.

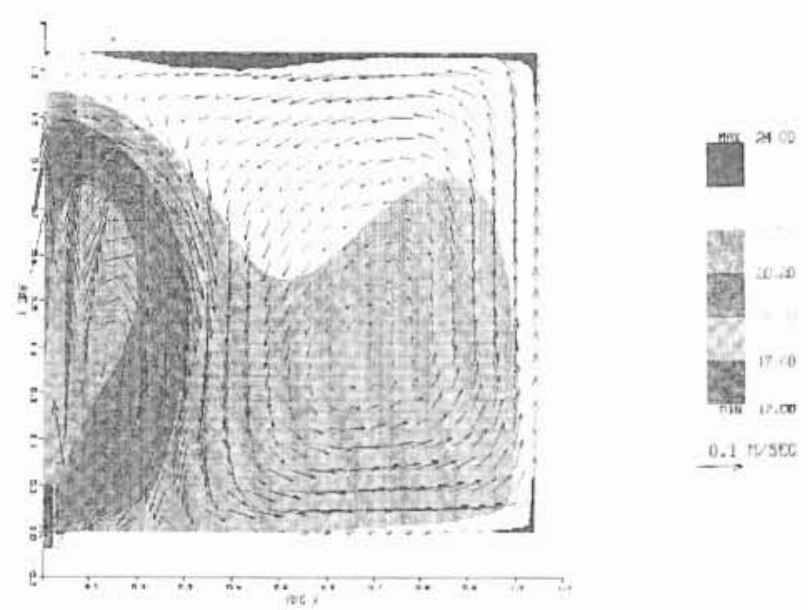

7. Champs de température et de vitesse pour le cas 1 .

\section{Profils verticaux de vitesse horizontale (fig. 8 ) :}

Mis à part le profil $X=0,2 \mathrm{~m}$, les vitesses horizontales sont parfaitement prédites par N3S.

Pour $X=0,2 \mathrm{~m}$, un décalage entre les deux courbes est constaté. Pour le calcul, la tête du jet s'affaisse beaucoup plus tôt $(Y=0,7 \mathrm{~m})$ que dans les mesures $(Y=1 \mathrm{~m})$. Cet affaissement est dû à la conjonction des forces de gravité négatives et de l'entraînement surestimé par N3S. L'effet du plafond semble en revanche négligeable.

Pour $X=1,023 \mathrm{~m}$, on note un pic de vitesse horizontale expérimentale dans le coin supérieur droit de la cavité qui n’apparaît pas dans les calculs.

\section{Profils horizontaux de vitesse verticale (fig. 9) :}

Dans l'ensemble, la vitesse verticale est correctement représentée par le code.

Pour $Y \leq 0,7 \mathrm{~m}$ on remarque cependant que le jet $\mathrm{N} 3 \mathrm{~S}$ est plus large que le jet expérimental. En effet la zone critique de basculement entre les deux tourbillons se situe 

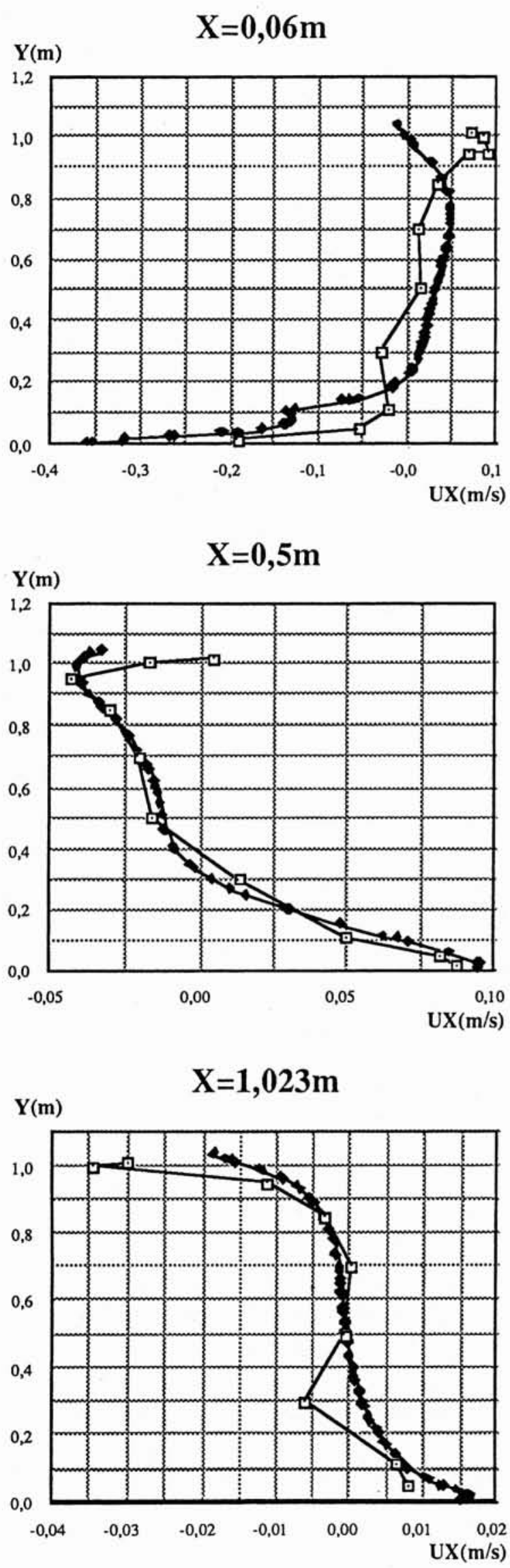

$$
\mathrm{X}=\mathbf{0 , 2 m}
$$

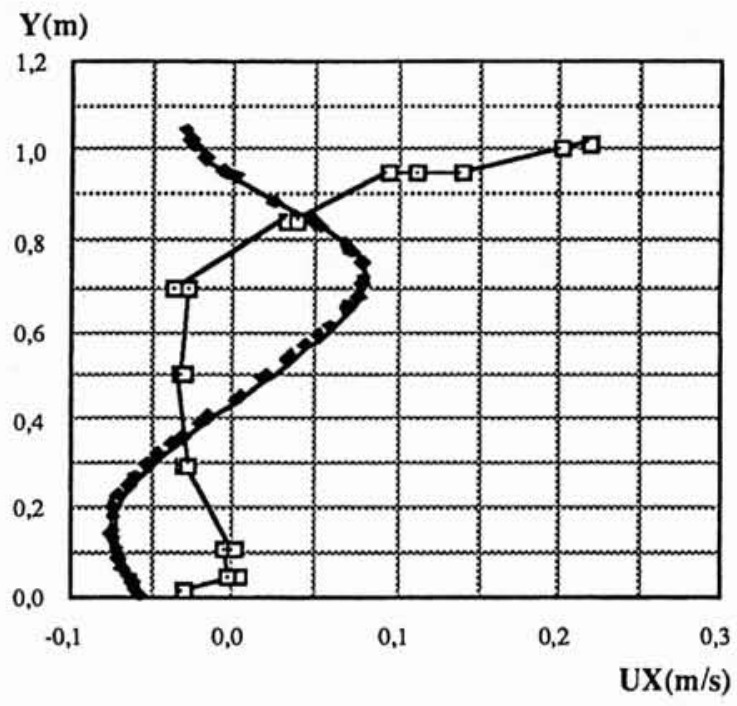

$$
\mathrm{X}=\mathbf{0 , 8 m}
$$

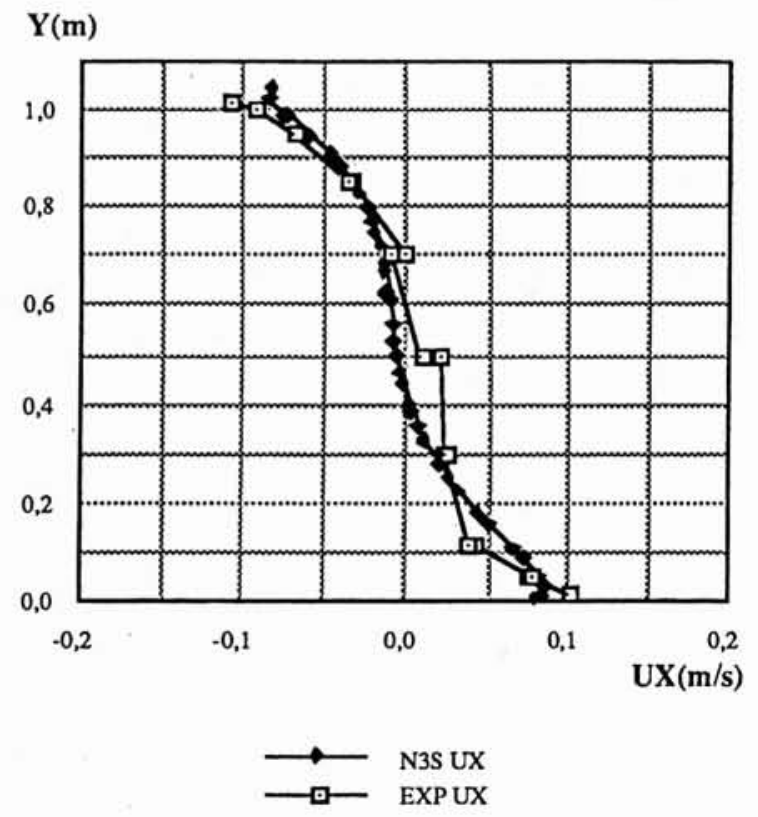

8. Profils verticaux de vitesse horizontale pour le cas 1 . 

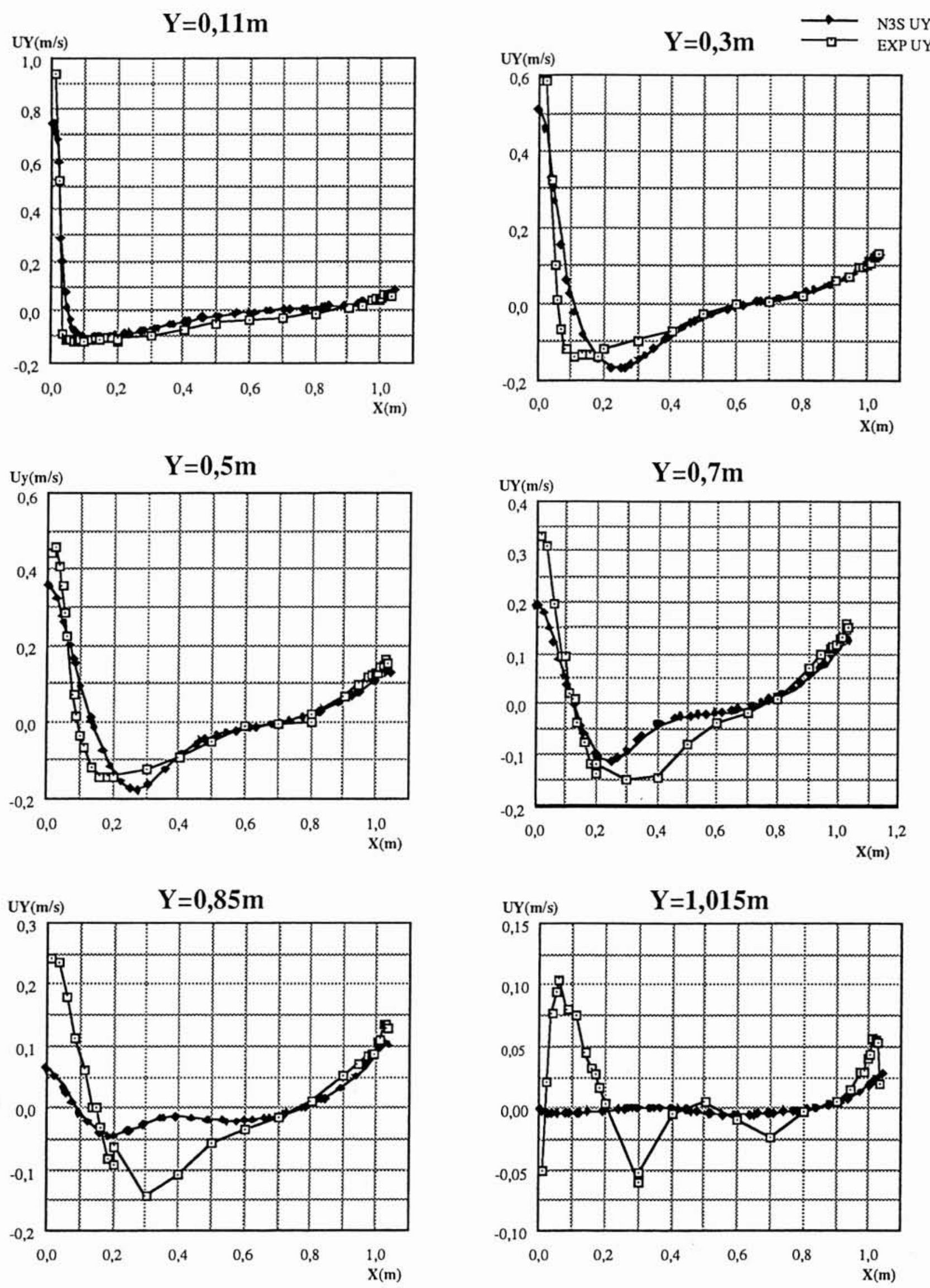

9. Profils horizontaux de vitesse verticale pour !e cas $\mathbf{1}$. 
à $X=0,18 \mathrm{~m}$ environ pour les expériences contre $X=0,23 \mathrm{~m}$ environ pour les calculs.

Au voisinage du plafond, la vitesse verticale N3S est quasiment nulle, le gradient est plat, alors que dans les expériences on constate un pic de vitesse verticale audessus du jet. En effet, dans les calculs, l'air stagnant le long du plafond n'est pas perturbé par le jet.

De façon générale, en ce qui concerne les vitesses, le calcul fournit des informations quantitatives très satisfaisantes. Les dimensions du jet sont en revanche prédites avec moins de précision : le jet N3S est moins haut et plus large que le jet expérimental. Cet écart est sans incidence sur la vitesse au centre et dans la partie droite de la cavité.

L'annexe B comparant les résultats de la corrélation générale de jet en milieu libre aux résultats expérimentaux enregistrés ici confirme l'indépendance de l'écoulement en sortie d'émetteur vis-à-vis du reste de la cellule. On retrouve en effet l'évolution linéaire du rayon du jet et la constance du coefficient d'entraînement $\alpha$ lié à l'hypothèse de longueur de mélange. Cependant l'évolution de la vitesse du jet ne suit pas tout à fait une loi classique de jet anisotherme en puissance $-1 / 2$ de la cote. Les effets de la poussée d'Archimède négative et des forces de frottement de l'air le long de la paroi de la cellule semblent en accentuer le ralentissement. Comme dans le cas du chauffage par convecteur, une étude complémentaire serait nécessaire pour en établir une représentation fiable.

Profils de température (fig. 10) :

Les faibles gradients verticaux de température sont bien représentés par le code.

On constate en revanche une déviation systématique de $1{ }^{\circ} \mathrm{C}$ à $2{ }^{\circ} \mathrm{C}$ entre les températures mesurées et calculées. N3S sous-estime la température sur l'ensemble de la cavité. L'annexe $\mathrm{A}$ confirme que cet écart est dû à la sousestimation du coefficient d'échange local. Cette observation est cohérente avec les résultats enregistrées sur les configurations de chauffage [2], bien qu'amplifiée par le fait que l'affaiblissement du panache surestimé par N3S modifie radicalement les conditions de température au voisinage du plafond et provoque un court-circuit plus important de l'air rafraîchi par la bouche d'extraction.

Par ailleurs, les profils horizontaux de température montrent que la zone refroidie prédite par N3S est plus large que celle observée. De même, les gradients de température calculés sont plus faibles que ceux mesurés à la frontière du jet. Ces phénomènes confirment l'hypothèse émise lors de l'analyse des champs de vitesse: N3S surestime dans cette configuration le mélange du jet et de l'air ambiant.

Enfin, dans le haut de la cavité, le long du plafond : les courbes de température prédites et mesurées sont très différentes. Cela s'explique par l'affaissement du jet surestimé par N3S.

3.3.2 Influence du profil de vitesse sur les dimensions du panache

Profils de vitesse (fig. 12, 13) :

Le panache obtenu avec un profil plat (cas 2) est lé- gèrement plus étroit que pour le profil expérimental. L'écart pour le troisième profil n'est pas visible.

\section{Profils de température (fig. 14, 15):}

De même que pour les vitesses, on note peu de différences concernant les champs de température entre les trois simulations.

On conclut donc que le profil de vitesse à la sortie du climatiseur, pour un soufflage vertical, n'a pas d'incidence notable sur l'irrigation de la cellule et son rafraîchissement.

\section{IV $\square$ CONCLUSION}

Cette qualification du code N3S version 3.1 menée sur une configuration de climatisation confirme les résultats obtenus précédemment sur des configurations de chauffage [2]. Les modèles physiques (modèle de turbulence standard $k \sim \varepsilon$, approximation de BouSSINESQ et couches limites aux parois représentées par une loi logarithmique) et les méthodes numériques (résolution du problème de STOKES par l'algorithme de CHORIN et maillage P1-isoP2) sont identiques à ceux mis en cuvre en 1993.

En termes de performance numériques on retrouve un rythme de $0,18 \mathrm{~s} / 1000$ nœuds et par pas de temps (cette valeur était de $0,11 \mathrm{~s}$ pour les simulations de chauffage), ce qui nous conduit à 3000 secondes de CPU de CRAY Y-MP pour 4000 itérations correspondant à 400 secondes de temps réel, durée nécessaire à l'établissement du régime permanent en thermique.

En termes de précision, on retrouve une très bonne prédiction du champ de vitesse et une estimation moins précise du champ de température. La sous-estimation du transfert de chaleur aux parois, sans doute accentuée par l'affaiblissement du jet surestimé par N3S, se traduit ici par une température trop faible d'environ $1,8^{\circ} \mathrm{C}$. Néanmoins, de même que pour les configurations de chauffage, l'absence de gradient vertical de température est simulé convenablement par le calcul.

En revanche, plus localement, des écarts parfois importants ont été relevés. En particulier, N3S surestime la largeur du jet et son interaction avec l'écoulement général. Cela se traduit premièrement par un affaiblissement plus marqué de celui-ci qui n'atteint pas le plafond de la cellule contrairement à ce qui a été observé expérimentalement. Deuxièmement, le court-circuit de l'air rafraîchi par la bouche d'extraction ainsi surestimé modifie fortement l'efficacité globale du climatiseur. L'utilisation directe des résultats de N3S peut donc conduire à une appréciation erronée du confort apporté.

L'annexe B montre qu'une corrélation simple peut être établie pour décrire l'écoulement au-dessus de l'émetteur. Cette corrélation est proche de celle d'un jet plan en milieu infini isotherme. En particulier, la loi de Morton est bien vérifiée, à savoir: l'entraînement ne dépend que de la vitesse du jet et lui est proportionnel. Cela confirme que le jet est très indépendant du reste de l'écoulement. Une simulation basée sur un modèle zonal est donc envisageable pour cette configuration. Il est à noter cependant que l'effet de la poussée d'Archimède à contre-courant et le frottement de l'air le long de la paroi de la cellule modifient l'évolution de la vitesse du jet qui ne suit pas une loi 

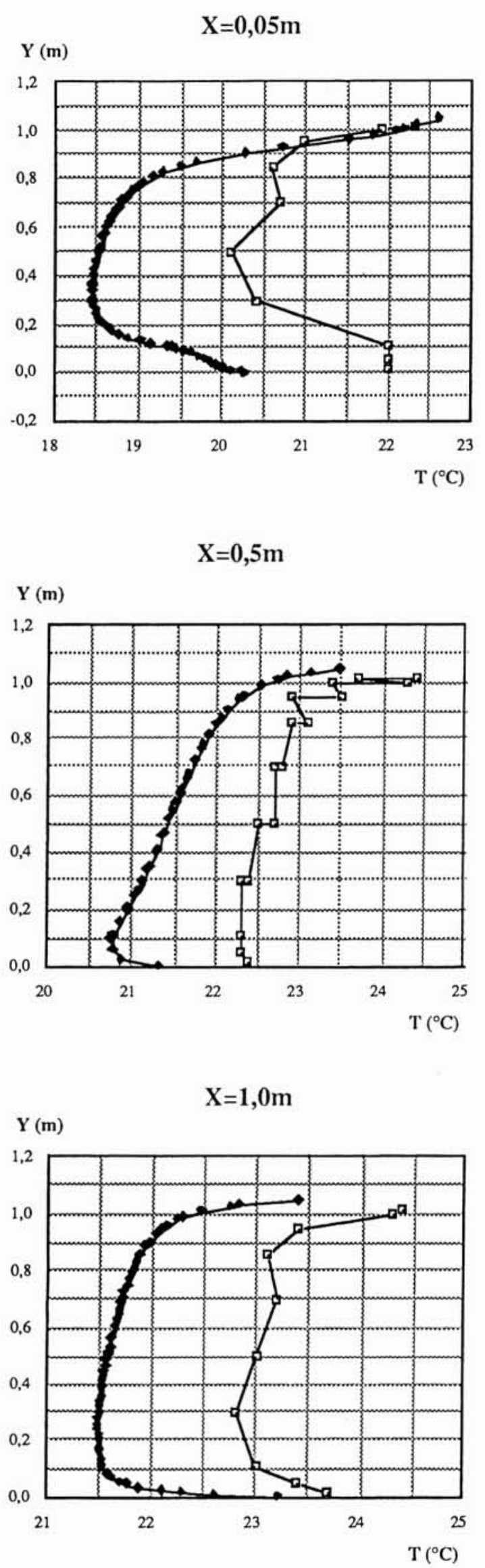

10. Profils verticaux de température pour le cas 1 .
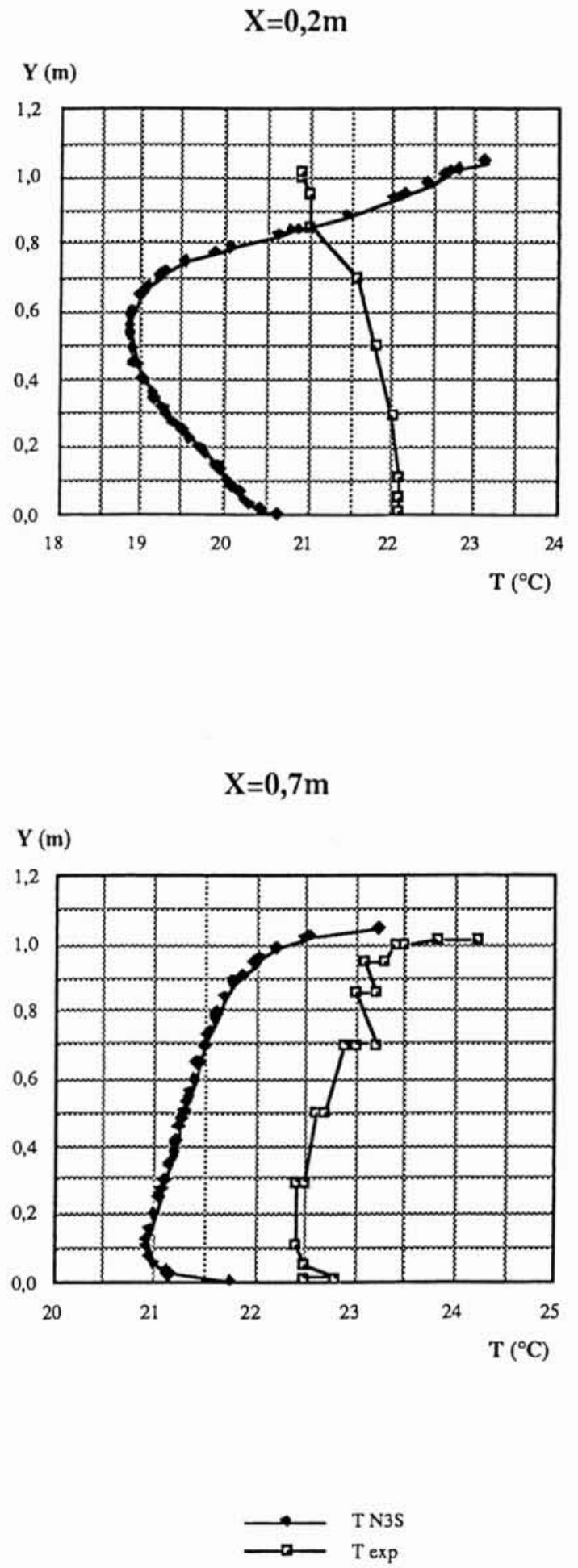
D. MANZONI, F.-X. RONGÈRE
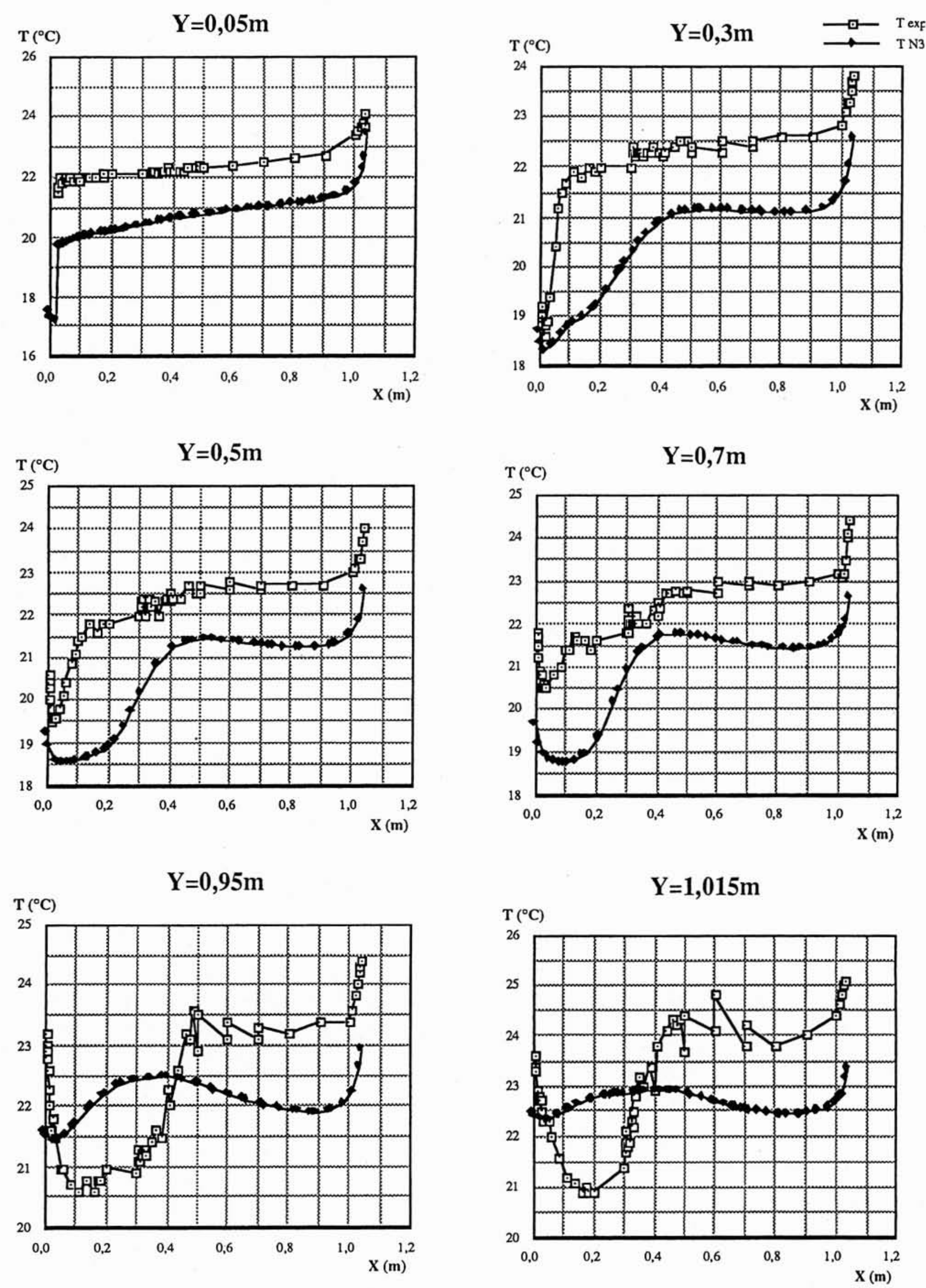

11. Profils horizontaux de température pour le cas 1 . 
SIMULATION AÉRAULIQUE D'UNE CELLULE CLIMATISÉE
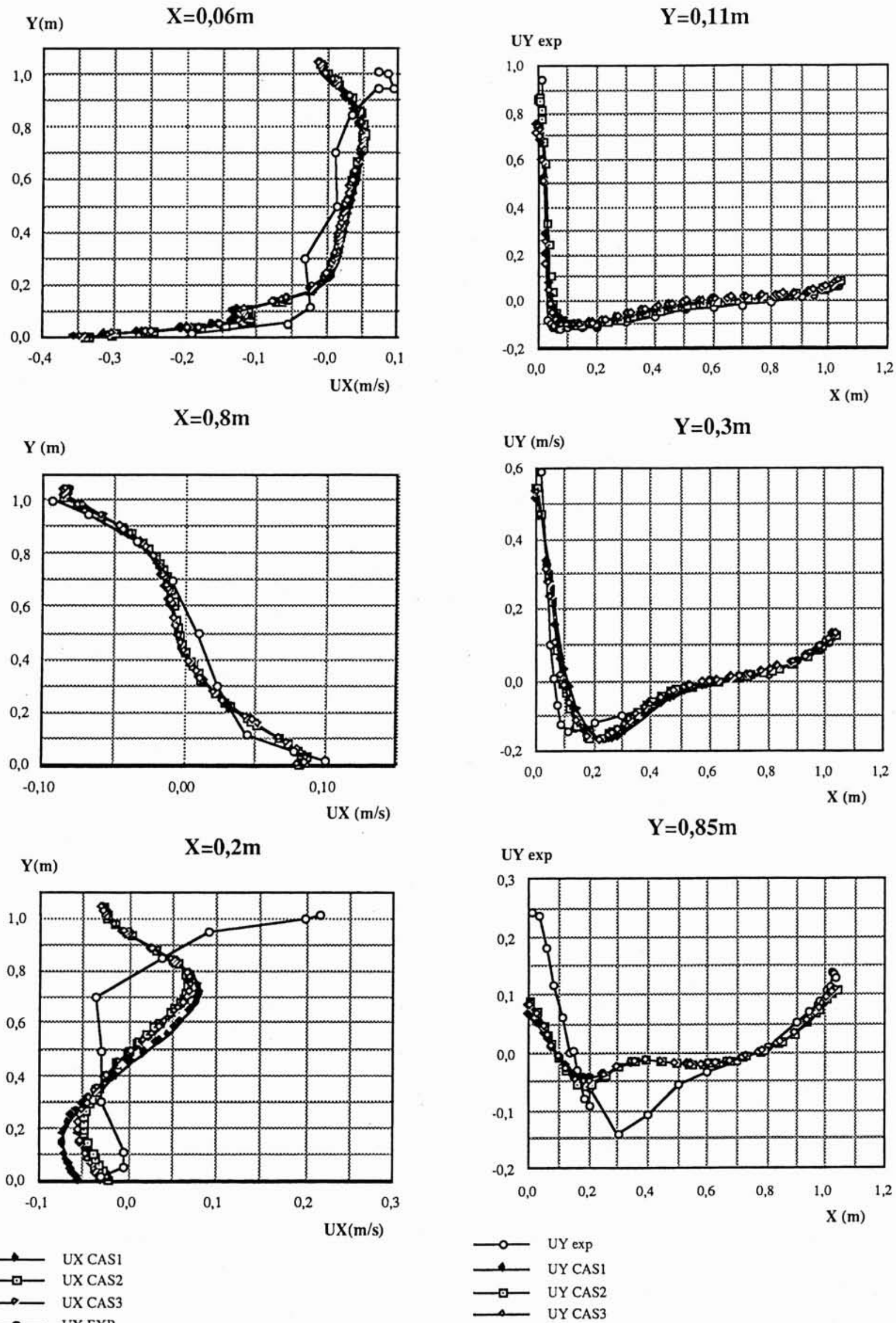

12. Profils verticaux de vitesse horizontale, cas 1-2-3.

13. Profils horizontaux de vitesse verticale, cas 1-2-3. 

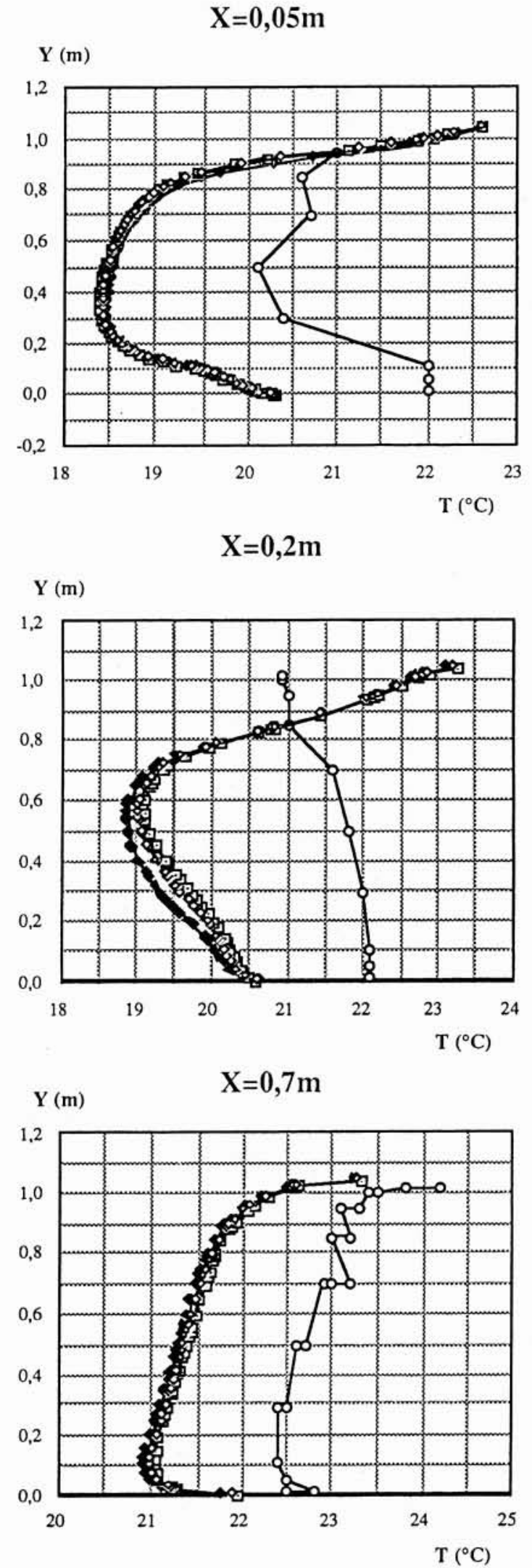

$\begin{array}{ll}\longrightarrow- & \text { UY exp } \\ \longrightarrow & \text { UY CAS1 } \\ \square- & \text { UY CAS2 } \\ \square & \text { UY CAS3 }\end{array}$

14. Profils verticaux de température, cas 1-2-3.
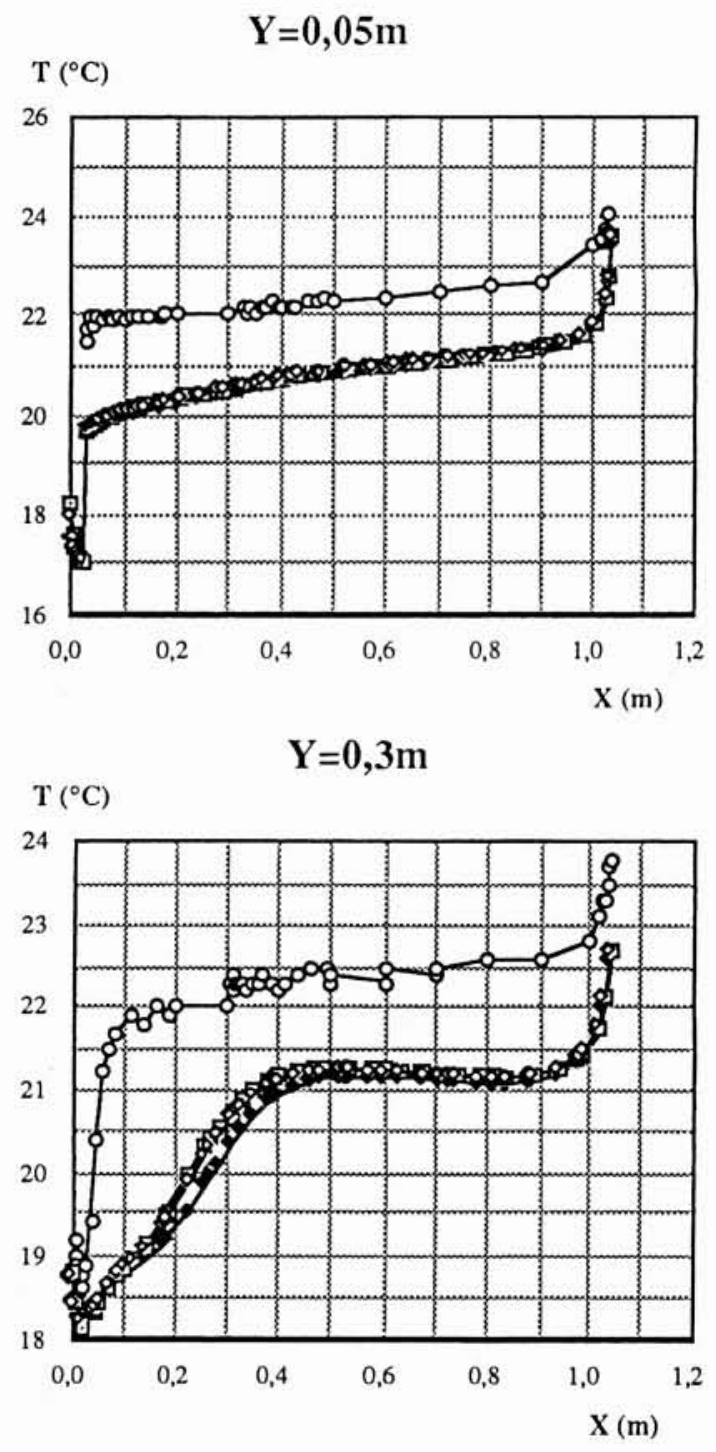

$$
\mathrm{Y}=0,7 \mathrm{~m}
$$

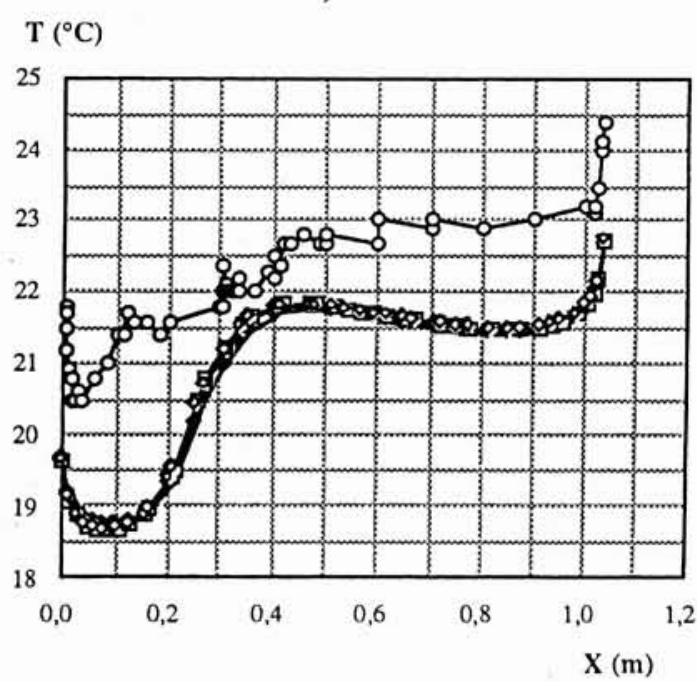

$\begin{array}{ll}\longrightarrow & \text { TEXP } \\ \longrightarrow & \text { TCAS1 } \\ \longrightarrow- & \text { TCAS2 } \\ \longrightarrow- & \text { TCAS3 }\end{array}$

15. Profils horizontaux de température, cas 1-2-3. 
classique. Comme dans le cas des convecteurs, une étude spécifique serait nécessaire pour préciser ces premiers résultats.

Enfin, ces simulations ont permis de démontrer que le profil de la vitesse de soufflage, lorsqu'elle est verticale, n'a pas d'incidence notable sur l'écoulement et le champ de température.

Ces conclusions confirment l'intérêt et les limitations du logiciel N3S pour l'analyse des écoulements dans les locaux chauffés ou rafraîchis. Il constitue en particulier un appui solide pour les modèles intégraux que nous développons. Son utilisation directe en qualification de confort doit en revanche être faite avec prudence du fait notamment de son imprécision sur le champ de température.

\section{Références}

[1] FANGER P.O. (1972). - Thermal comfort. Analysis and applications environmental engineering. Mac Graw-Hill, New York.

[2] BLAY D. (1993). - Etude expérimentale d'écoulements convectifs dans une configuration de climatisation des locaux. Rapport L.E.T., novembre 1993 de I'ENSMA (Poitiers).

[3] MANZONI D., RONGĖRE F.X. - Simulation aéraulique d'une cellule chauffée et ventilée. Rapport EDF HT-31/93/018/A.

[4] DeLENNE B. - Calculs par éléments finis de l'écoulement 2D dans une pièce d'habitation. Rapport EDF HT-31/91/022/A.

[5] DELENNE B. - Aéraulique de l'habitat. Calculs par éléments finis d'écoulements bidimensionnels. Rapport EDF HT31/92/005/A.

[6] LıONNET B. - Etude comparative de logiciels de mécanique des fluides en aéraulique. Rapport EDF HE-11/93/002.

17] RoDI W. (1982). - Turbulent jets and plumes. Pergamon Press.

\section{Annexe A : bilan sur la cavité Evaluation des coefficients d'échange}

Le flux perdu par les parois, noté $\varphi_{p}$, est égal à la somme des flux entrant moins la somme des flux sortant (fig. 16), donc :

$$
\varphi_{p}=\rho_{e} Q_{e} C p_{e} T_{e}-\rho_{s} Q_{s} C p_{s} T_{s}=h \cdot s\left(T_{m i l}-T_{p}\right)
$$

avec :

- $T_{e}$ : La température du flux froid entrant, de capacité thermique $C p_{e}$, de vitesse débitante $U_{j}$, de débit $Q_{e}$, et de masse volumique $\rho_{c}$.

- $T_{s}$ : La température au voisinage de la sortie d'air, de capacité thermique $C p_{s}$, de débit $Q_{s}$, et de masse volumique $\rho_{s}$.

- $T_{m i l}$ : La température au milieu de la cavité.

$-T_{p}$ : La température de parois.

- $s$ : La surface des parois.

Le tableau ci-dessous compare ces valeurs obtenues expérimentalement et par calcul :

\begin{tabular}{|l|r|r|}
\hline Grandeurs & Mesures & Calcul \\
\hline$\rho e\left(\mathrm{~kg} / \mathrm{m}^{3}\right)$ & \multicolumn{2}{|c|}{1,217} \\
\hline$T_{e}\left({ }^{\circ} \mathrm{C}\right)$ & \multicolumn{2}{|c|}{17,0} \\
\hline$C p_{e}$ et $C p_{s}(\mathrm{~J} / \mathrm{kg} . \mathrm{K})$ & \multicolumn{2}{|c|}{1006} \\
\hline$Q_{e}\left(\mathrm{~m}^{2} / \mathrm{s}\right)$ & \multicolumn{2}{|c|}{0,0168} \\
\hline $\mathrm{s}(\mathrm{m})$ & \multicolumn{2}{|c|}{4,16} \\
\hline$T_{p}\left({ }^{\circ} \mathrm{C}\right)$ & \multicolumn{2}{|c|}{26,7} \\
\hline$\rho_{s}\left(\mathrm{~kg} / \mathrm{m}^{3}\right)$ & 1,198 & 1,205 \\
\hline$T_{s}\left({ }^{\circ} \mathrm{C}\right)$ & 21,8 & 19,9 \\
\hline$Q_{s}\left(\mathrm{~m}^{2} / \mathrm{s}\right)$ & 0,0171 & 0,0170 \\
\hline$T_{m i l}\left({ }^{\circ} \mathrm{C}\right)$ & 22,5 & 21,3 \\
\hline$\varphi_{p}(\mathrm{~W} / \mathrm{m})$ & $-98,7$ & $-59,6$ \\
\hline$h\left(\mathrm{~W} / \mathrm{m}^{2} . \mathrm{K}\right)$ & 5,65 & 2,66 \\
\hline
\end{tabular}

On remarque ici que, premièrement, le coefficient d'échange global obtenu à partir des valeurs expérimentales est voisin de celui obtenu dans les mêmes conditions pour les configurations de chauffage, notamment par convecteur: $h_{\text {mes }}=5,64 \mathrm{~W} / \mathrm{m}^{2} . \mathrm{K}[2]$. Cette valeur généralement admise en thermique du bâtiment semble donc applicable en climatisation comme en chauffage.

Deuxièmement, alors que l'écart de température entre

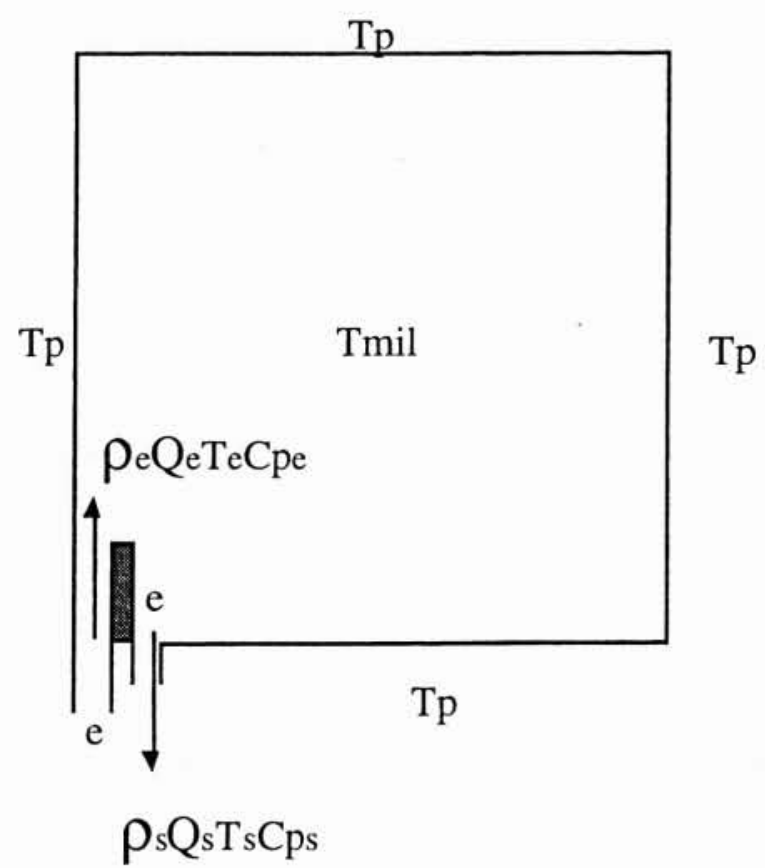

16. Bilan d'énergie sur la cavité. 
mesures et calcul est le même ici qu'en chauffage par convecteur $\left(\Delta T_{m i l}=1,8^{\circ} \mathrm{C}\right)$, l'écart sur les coefficients d'échange globaux est beaucoup plus grand $\left(h_{c a l} / h_{\text {mes }}=0,48 \mathrm{ici}\right.$, contre $h_{c a l} / h_{\text {mes }}=0,71$ en chauffage par convecteur [2]). Cette différence peut être due soit à une erreur sur la couche limite plus forte en climatisation qu'en chauffage soit, plus sûrement, à l'effet de l'affaissement du panache surestimé par N3S ici, qui modifie radicalement les conditions de température au voisinage du plafond et provoque un court-circuit plus important de l'air rafraîchi par la bouche d'extraction. En effet $\left(T_{\text {mil }}-T_{s}\right)_{c a l}=1,4^{\circ} \mathrm{C}$ alors que $\left(T_{m i l}-T_{s}\right)_{m e s}=0,7^{\circ} \mathrm{C}$; cet écart représente presque $25 \%$ du bilan énergétique global de la cellule.

Notons enfin, que ce lien différent entre coefficient d'échange et température au centre de la pièce provient du fait que dans la configuration de climatisation la sousestimation de la température dans la cellule se traduit par un bilan d'énergie $\varphi_{p}$ plus faible et un écart de température $T_{m i l}-T_{p}$ plus fort alors qu'en chauffage cet effet s'applique dans le même sens pour le bilan d'énergie et pour l'écart de température.

\section{Annexe B : Etude du jet expérimental}

Cette étude a pour objet de comparer les corrélations classiques de jet en environnement infini avec des résultats expérimentaux mesurés en enceinte.

Le jet d'air froid est dirigé selon l'axe vertical (axe des ordonnées $y$ ). Sur cet axe la vitesse verticale du jet est maximale : elle est notée $V_{\max }(Y)$. Pour une hauteur $Y$ donnée, la largeur du jet est notée $B(Y)$.

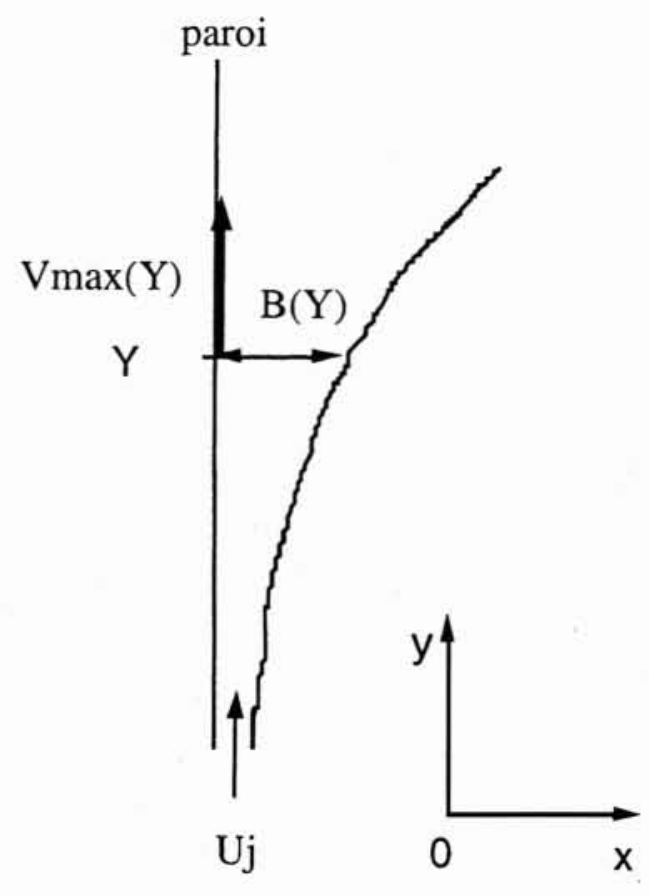

17. Allure du jet expérimental.
Le jet expérimental est mis en mouvement par un moment initial avec des forces d'Archimède défavorables. On détermine la vitesse maximale et la largeur du jet en fonction de la cote $y$.

\begin{tabular}{|c|c|c|}
\hline$Y(m)$ & $V_{\max }(\mathrm{m} / \mathrm{s})$ & $B(m)$ \\
\hline 0,110 & 0,939 & 0,031 \\
0,300 & 0,587 & 0,061 \\
0,500 & 0,445 & 0,093 \\
0,700 & 0,334 & 0,126 \\
0,850 & 0,244 & 0,150 \\
\hline
\end{tabular}

B.1 Etude de la largeur du jet en fonction de la hauteur

La figure 18 traduit l'évolution du rayon du jet en fonction de la cote.

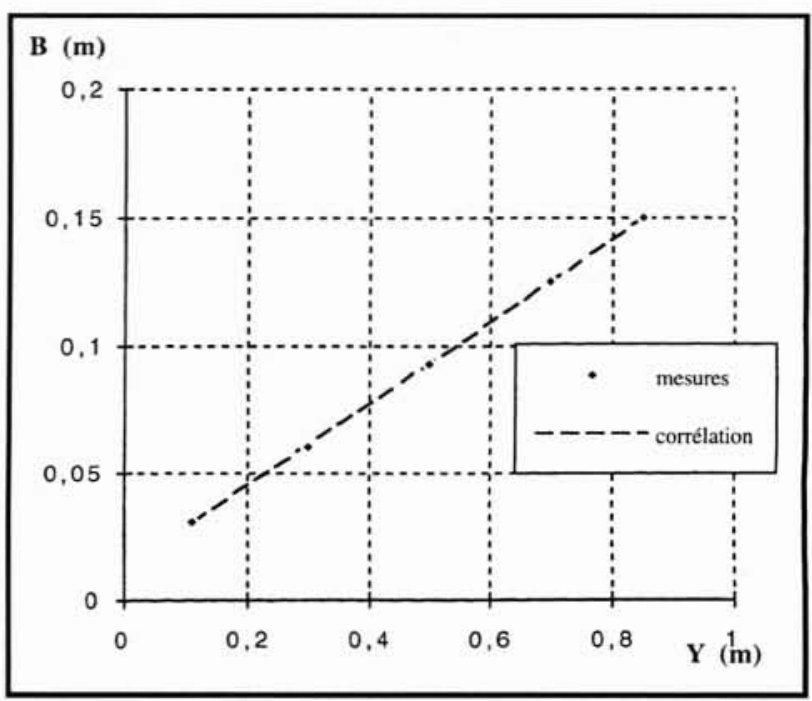

18. Evolution de la largeur du jet en fonction de la cote.

La corrélation correspondante vaut :

$$
B=0,012+0,161 \cdot Y\left(r^{2}=1,00\right)
$$

On vérifie bien que la largeur du jet varie linéairement en fonction de la cote $y$. L'approximation entre les points expérimentaux et la droite est de plus très satisfaisante. Cela démontre que le jet suit la loi de Morton et al. reposant sur une hypothèse de longueur de mélange:

$$
\frac{\mathrm{d}\left(B \cdot V_{\max }\right)}{\mathrm{d} y}=\alpha \cdot V_{\max }
$$

où $\alpha$ est un coefficient d'entraînement constant. 


\section{SIMULATION AÉRAULIQUE D'UNE CELLULE CLIMATISÉE}

B.2 Etude de la vitesse maximum dans le jet en fonction de la hauteur

De même la figure 19 traduit l'évolution logarithmique de la vitesse maximale du jet en fonction de la largeur du jet.

La corrélation est de la forme:

$$
\ln \left(V_{\max }\right)=-1,35-0,618 \ln (Y)\left(r^{2}=0,953\right) .
$$

Or, pour un jet pur plan, l'équation de quantité de mouvement impose:

$$
\frac{\mathrm{d}\left(B \cdot V_{\max ^{2}}\right)}{\mathrm{d} y}=0 .
$$

Ce qui conduit, par analyse dimensionnelle, à $V_{\max }$ proportionnelle à $y^{-0.5}$. L'évolution mesurée est donc proche d'un comportement de jet libre. L'effet de la poussée d'Archimède négative et le frottement le long de la paroi sont sans doute à l'origine de l'écart observé.

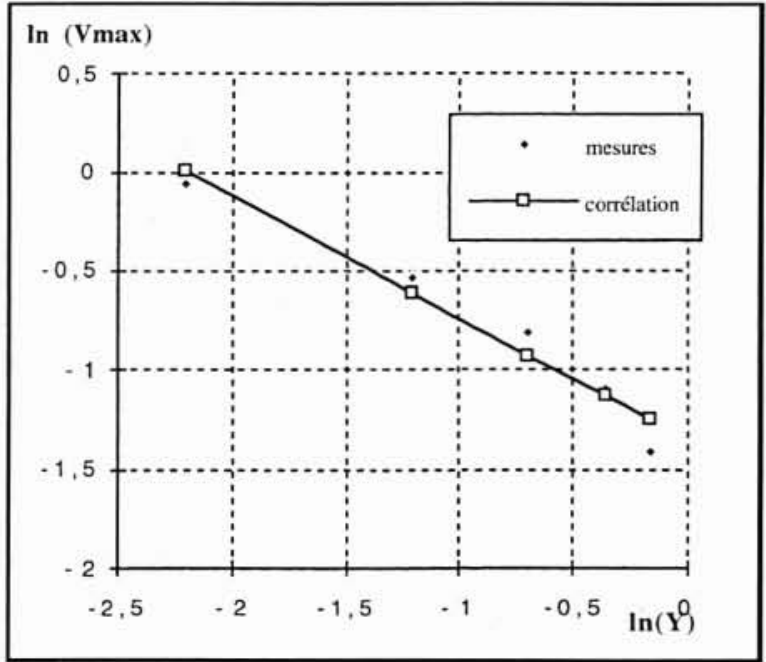

19. Evolution logarithmique de la vitesse maximale du jet en fonction de la largeur du jet. 